\title{
Symbolic comparison of color similarity
}

\author{
JOHN TE LINDE and ALLAN PAIVIO \\ University of Western Ontario, London, Ontario N6A 5C2 Canada
}

This study investigated symbolic comparison of color similarity using a triplet paradigm. Results showed that the time to choose which of two color samples is more similar to a color name was a function of several measures of distance between the samples relative to the focal color for the category name. Since colors appear to be represented in memory only as names and images and not as abstract entities such as features or propositions, these results provide support for models of symbolic comparison that assume that items are stored and compared as mental analogs.

Recent research on the manner in which our knowledge of the world is represented in long-term memory has made use of a paradigm that involves comparative judgment of symbolic information. The participants in these experiments are shown a pair of stimuli, such as names or pictures of familiar objects or animals, and their task is to decide which of the two is, for example, larger in real life.

The basic finding from these experiments is a systematic decrease in the time required to choose between the two symbols as the psychological distance between them increases. For example, Moyer (1973) found that the reaction time (RT) to choose the larger of two named animals decreased as the difference in rated sizes of the animals increased. These results are similar to those obtained when subjects are asked to make size comparisons among physical stimuli actually presented (Curtis, Paulos, \& Rule, 1973).

Several findings within a variety of symbolic comparison tasks permit strong inferences concerning the nature of symbolic information in long-term memory. Moyer (1973) suggested that his subjects made some kind of internal "psychophysical judgment" among analog memory representations. Such representations store our knowledge of the world in a form that is highly isomorphic with or analogous to perceptual knowledge (Attneave, 1972; Cooper \& Shepard, 1973). They "contain" information that bears a continuous (analog) relation to the perceptual information aroused by the perceptual objects themselves. Paivio (1975) proposed that these analog representations may take the form of

This research was supported by a postgraduate scholarship to the first author and Grant A0087 to the second author from the National Research Council of Canada. The authors thank lan Spence for his help in scaling the data. They also thank Keith Holyoak and an anonymous reviewer for comments on an earlier version of this paper. Portions of this paper were presented at the annual meeting of the Canadian Psychological Association, Ottawa, June 7-9, 1978. Requests for reprints should be sent to John te Linde, Department of Psychology, University of Western Ontario, London, Ontario N6A 5C2, Canada. visual images. According to dual-coding theory (Paivio, 1975, 1978), two independent but interconnected systems exist in memory for retaining knowledge of the world. Perceptual information, such as the sizes of objects, is stored in a nonverbal image-processing system. Linguistic information, on the other hand, is stored separately in a verbal processing system. According to the model, subjects in a size comparison task would make their judgments by first converting the object names to analog (possibly image) codes and then comparing these representations.

Paivio (1975, 1978) recently tested dual-coding theory and other theories that assume a more abstract (essentially linguistic) data base for symbolic comparisons. In one series of experiments (Paivio, 1975), Moyer's (1973) basic finding was extended to include size comparisons of objects from an infinite rather than a finite set, comparisons within and across conceptual categories, and comparisons using pictures and words as stimuli. In addition to verifying the symbolic distance effect, these findings demonstrated (1) that decision times are not determined by subjects' use of overlearned verbal labels (since the items were never presented more than once to a subject), (2) that RTs did not vary as a function of category membership, (3) that pictures of objects resulted in consistently faster comparison times than did object names, and (4) that it is possible to induce a Stroop-type conflict when pictured size difference is incongruent with memory size difference.

Semantic network and propositional models of longterm memory cannot easily explain such findings (see discussions by Banks, 1977; Paivio, 1975). Such approaches, for example, are seriously constrained by the finding that category membership per se has no effect on size comparisons. More importantly, propositional theories predict no distance effect or one opposite to that obtained; that is, the smaller the difference, the shorter the connecting path between the two items, and the faster the predicted decision time. Dual-coding theory, on the other hand, is able to account for the results. Subjects simply retrieve and compare the items 
as mental analogs without regard for class membership or verbal associative relations between the object and the comparative. The picture-word differences in $\mathrm{RT}$ are predicted from the theory since the analog/image system is assumed to be accessed more directly given pictures rather than words as stimuli. The assumption that manipulations within the visual memory system may involve visual processes readily predicts the Strooptype conflict obtained with pictured but not named stimulus items.

Reported here is an experiment designed to extend the symbolic comparison paradigm to judgments of color similarity. Color possesses several unique perceptual and semantic attributes that are of particular theoretical interest when color is used as a variable in symbolic comparisons. First, colors appear to be examples of items that are represented in semantic memory only as names and images. Katz and Postal (1964), for example, use color distinctions in linguistics as an example of categories that are based purely on perception and not codable by linguistic features. Shepard (1964) states that colors are examples of "unitary" stimuli that tend to be "reacted to as homogeneous unanalyzable wholes" (p. 59); such stimuli are distinct from those that can be analyzed into perceptually discrete components or properties (e.g., geometric figures). It is reasonable to assert, then, that much of our knowledge of the psychological relations among colors is based on perception and memory images and not on language. Indeed, Shepard and Cooper (Note 1) found that congenitally blind subjects and a pure rod monochromat, in contrast to normally sighted subjects, gave highly variable judgments when asked to judge similarity between pairs of color names. These data and the preceding statements imply that it would be difficult to explain how similarity comparisons between named colors could be made from a discrete abstract or propositional data base representing color in semantic memory.

A study of comparative judgments involving color is interesting for a second reason. Whereas previous research in comparative judgment has involved bipolar linear continua such as size, friendliness, and so on, comparison of hue involves an underlying nonpolar circular dimension, namely, the hue circle. This dimension requires that, instead of comparing items with respect to a polar reference point implied by the instructions in previous tasks, subjects must be given an explicit point of reference. By using a triplet paradigm where subjects are asked to judge which of two colors is more similar to a named color, for example, the reference point (i.e., the named color) is explicitly given and can be varied from trial to trial. These features provide a new potential source of evidence for evaluating comparison models. For example, the color circle would eliminate effects of the polar reference present in linear continua. A verbal associative approach might explain the symbolic distance effect in terms of a re- sponse bias resulting from frequent associations between the verbal labels for the poles of a linear continuum and items falling near those extremes. On the color circle, however, similarity relations between colors cannot be coded with reference to any fixed point or pole. Obtaining a symbolic distance effect by varying the psychological similarity of colors presented for comparison would definitively rule out accounts of the function in terms of verbal associative habits between items and points on the continuum.

The present study was an attempt to determine whether a symbolic distance effect can be obtained for judgments of color similarity between a color name and two color chips of varying distance from the color name on the hue circle.

\section{METHOD}

\section{Stimuli}

The stimuli were selected from the color chart used by Berlin and Kay (1969) in their study of color categorization across languages. This chart is composed of 320 Munsell color chips of 40 subjectively equally spaced hues and $8 \mathrm{deg}$ of brightness, all at maximum saturation. The entire array forms a complete hue circle. The stimuli used in the present experiment were seven basic color names (red, orange, yellow, green, blue, purple, and pink) and 21 color chips from the chart. These color chips were 7 chosen as the best exemplars of the color names in the Berlin and Kay study and 14 chips located toward the boundaries of the categories denoted by the color names. Thus, each color name was represented by one focal and two peripheral color chips. For those cases on the Berlin and Kay chart where more than one focal color chip existed for the color name, the chip most central for the category on the hue dimension or the chip nearest the midpoint on the brightness dimension was selected. The color chips and the color categories used are depicted in Figure 1.

From the chart, each of the seven focal color chips can be specified in terms of the number of perceptual steps that separates it from the other focal chips. For example, the focal color chip for yellow has a distance ${ }^{1}$ of 22 visual steps from the focal color chip for blue, while a shorter distance of 8 steps separate the yellow and orange focal chips. In this manner, the shortest (i.e., progressing in the shortest direction around the hue circle depicted by the chart) distance between all possible pairs of the seven focal color chips was measured. This yielded a range of distance measures from 2 steps for red/orange to 22

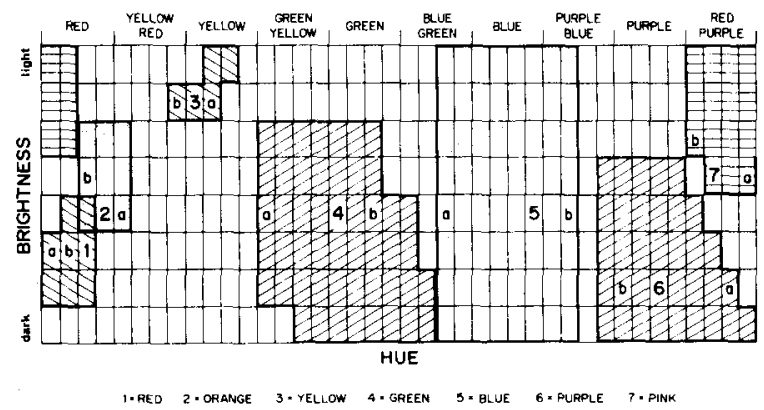

Figure 1. Color chart depicting the color chips (focal $=$ numerals, peripheral $=$ letters) and color categories used in constructing triplets (adapted from Berlin \& Kay, 1969). 
steps for yellow/blue. Each of the 14 peripheral color chips was also measured relative to the focal color chip for its category, yielding a range of distance measures from one to five steps.

Stimulus triplets were constructed from the distance measures described above. Each display consisted of a color name and two color chips of varying distance from the color name positioned on either side of the name. For example, the name "red" was combined with a red peripheral color chip and the focal color chip for yellow. Stimulus triplets were constructed in this manner for 189 combinations of color names and color chips; 27 triplets for each color name.

For each of these triplets, three indices of psychological distance were computed. For example, the green (chip)/red (name)/blue (chip) triplet yields a least distance of 13 steps, a subtractive distance of 3 steps, and a ratio distance of .81 . Least distance refers to the distance between the color name and the closer color chip. Subtractive distance is the distance remaining after the distance separating the color name and the closer color chip is subtracted from the distance separating the color name and the further color chip. Ratio distance is simply the ratio of the distance between the color name and the closer color chip to the distance between the color name and the further color chip. These distance measures (least, subtractive, and ratio) have been shown to be significant predictors of RT in other symbolic comparison tasks. Subtractive distance has been the usual measure in pair comparison tasks, such as judgments of relative size (e.g., Moyer, 1973; Moyer \& Bayer, 1976; Moyer \& Landauer, 1967). Parkman (1971), in a study requiring subjects to choose the larger of a pair of numbers, found that the numerical distance of the digit closer to zero was the best of a number of predictors of RT, including subtractive distance, or "split," as he termed it. The importance of ratio distance has been implicated in a number of studies as well. Paivio (1975), in a size comparison task, demonstrated that pairs of objects with larger size ratios (based on normative ratings of object sizes) produced faster decision times than pairs with small size ratios. Holyoak's (1978) reference point model for number comparisons is founded on the principle that RT is a linear function of a distance ratio of two compared numbers from a reference point. As a temporary compromise to the factorial structure of the present experiment, subtractive distance was used as the distance variable in constructing stimulus lists. Of the three, this measure permitted proper balancing of psychological distance values across the category and directionality variables included in the study (see later explanation of between-within category and uni-/ bidirectional triplets). The use of subtractive distance does not mean it was felt to be the best of the three distance predictors of $\mathrm{RT}$, only that it is one possible measure for demonstrating a symbolic distance effect in color comparison.

The 189 stimulus triplets yielded subtractive distances ranging from 0 to 21 hue steps. This range of values was sampled fairly equally within the 27 triplets for each color name, with the exception of distances greater than 14 steps. Since there were only 17 triplets with distances greater than 14 , these triplets were eliminated from further consideration, leaving 172 triplets with distances ranging from 0 to 14 steps. These triplets were collapsed into five groups. Group 1 included all those triplets having subtractive distances of 0,1 , and 2 ; Group 2 included all those triplets having subtractive distances of 3,4 , and 5; and so on through Group 5, which included those triplets with distances of 12,13 , and 14 .

A second variable included in the design was the directionality of the subtractive distance, that is, whether subtractive distance was obtained from measurements proceeding in the same direction around the color circle from the color name (unidirectional) or from measurements proceeding in opposite directions around the color circle from the color name (bidirectional). For example, the subtractive distance value for the yellow (chip)/red (name)/orange (chip) triplet is unidirectional, since the yellow/red and orange/red original distance values were obtained by counting steps in the same direction around the hue circle. On the other hand, the orange (chip)/red (name)/ purple (chip) subtractive distance is bidirectional, since the original distance values were obtained by counting in opposite directions around the hue circle. The stimulus triplets were further grouped into those involving a within-category comparison (i.e., those including a peripheral color chip from the same category as the color name) and those involving only comparisons across color categories (between-category comparisons). Examples of the stimulus triplets are given in Table 1.

Four different lists were constructed. Each list consisted of 60 different triplets including an equal number (12) from each of the five subtractive distance groups. Within each of these distance groups, eight triplets of the between-category type were included, with these split evenly between unidirectional and bidirectional triplets. Similarly, four triplets of the withincategory type were included in each of the five distance groups, with these split equally between unidirectional and bidirectional types. A triplet appeared only once in any one list, although some were repeated across lists. Four additional lists were constructed by reversing the left-right position of the color chips.

\section{Procedure}

For each triplet within the four basic lists, the color name was typed in Executive typeface capitals on the center of a $10.2 \times 15.3 \mathrm{~cm}(4 \times 6$ in.) neutral gray card. These cards were constructed so that two $1.5-\mathrm{cm}$ square color chips could be positioned $2 \mathrm{~cm}$ to the left and right of the color name.

The triplets were presented in a Gerbrands two-channel mirror tachistoscope. Luminance was equated as nearly as possible with that specified by the Munsell Color Company (1966) for color chip comparison. Each trial consisted of a verbal "ready" signal followed by a fixation field $(500 \mathrm{msec}$ duration). This was followed by exposure of the stimulus triplet for as long as the subject needed to respond. Five practice trials preceded presentation of the experimental items. All subjects understood that they were to press the button on the same side as the color chip more similar in color to the color name. RTs were recorded on a PDP-12 computer.

Four subjects were assigned to each of the four basic lists

Table 1

Examples of Triplets Used

\begin{tabular}{|c|c|c|c|c|c|c|c|}
\hline \multirow{2}{*}{$\begin{array}{l}\text { Color } \\
\text { Chip }\end{array}$} & \multirow{2}{*}{$\begin{array}{l}\text { Color } \\
\text { Name }\end{array}$} & \multirow[b]{2}{*}{ Color Chip } & \multicolumn{3}{|c|}{ Distance Measures } & \multirow[b]{2}{*}{ Directionality } & \multirow{2}{*}{$\begin{array}{r}\text { Category } \\
\text { Condition }\end{array}$} \\
\hline & & & Subtractive & Ratio & Least & & \\
\hline Purple & Orange & Pink & 4 & .64 & 7 & Uni- & Between \\
\hline Green & Red & Blue & 1 & .94 & 15 & $\mathrm{Bi}-$ & Between \\
\hline Orange & Yellow & Peripheral Yellow (b) & 7 & .13 & 1 & Uni- & Within \\
\hline Red & Green & Peripheral Green (a) & 11 & .27 & 4 & Uni- & Within \\
\hline Yellow & Blue & Purple & 13 & .41 & 9 & $\mathrm{Bi}-$ & Between \\
\hline Red & Purple & Peripheral Purple (b) & 7 & .22 & 2 & $\mathrm{Bi}-$ & Within \\
\hline Yellow & Pink & Red & 6 & .54 & 7 & Uni & Between \\
\hline
\end{tabular}


(two to each list and its left-right reversal). Triplets within each list were presented in random order, and the left-right positioning of the more similar color chip was also randomized over triplets. Each subject was presented his list twice, once in the forward and once in the reverse order. Thus an experimental session consisted of a color discrimination test followed by the first presentation of a list. The subject was then given a brief rest and asked to perform a simple rating task as part of another experiment. Finally, the subject was given the second presentation of the list.

\section{Subjects}

Sixteen subjects (aged 18-45 years) participated in the experiment, either for course credit (10) or for pay (6). All subjects were prescreened for color discrimination ability with the Farnsworth-Munsell 100-Hue Test (1957). All subjects tested were able to complete the experiment.

\section{RESULTS}

Initially, a 5 (distance groups) by 2 (within-/betweencategory comparisons) by 2 (directionality) by 2 (first vs. second presentation of lists) by 2 (left-right order) by 4 (lists) analysis of variance, with repeated measures on the first four factors, was carried out on the median correct RTs. The two or three triplets in each list with a subtractive distance of 0 from the Group 1 distance group were scored as correct regardless of the choice made, since both chips would be correct as defined by the color chart. The percentages of incorrect choices excluded from the analysis were $15.6 \%, 18.7 \%, 9.1 \%$, $8.6 \%$, and $5.7 \%$ for subractive distance Groups $1,2,3$, 4 , and 5 , respectively, collapsing over lists, directionality, and between-/within-category comparison conditions. Since the first vs. second presentation of a list had minimal direct and interactions effects in the analysis, this variable was dropped from further analyses.

The results showed significant main effects for subtractive distance groups $[\mathrm{F}(4,32)=36.8, \mathrm{p}<.001]$ and for between-/within-category comparison conditions $[F(1,8)=64.2, \quad p<.001]$. Separate ANOVAS for the between-and within-category comparisons condition both showed main effects of subtractive distance $[F(4,32)=15.6, p<.001$, and $F(4,32)=12.5, p<.001$, respectively]. The only other significant effects were a triple interaction of Directionality by Distance Groups by Between-/Within-Comparison Conditions $[F(4,32)=$ $3.4, \mathrm{p}<.05]$ and a quadruple interaction of Lists by Directionality by Distance Groups by Between-/WithinComparison Conditions $[\mathrm{F}(12,32)=2.4, \mathrm{p}<.05]$. Two considerations suggest that these interactions present no serious qualification of the distance group or of the between-/within-category comparison effect. First, the distance and category variables did not interact with each other, nor did they separately interact with lists or directionality. Second, the mean squares for the triple and quadruple interactions were .066 and .048 , re spectively, values that are much smaller than those for the distance group effect $\left(\mathrm{MS}_{\text {effect }}=.58\right)$ and the between-/within-category comparison effect $\left(\mathrm{MS}_{\mathrm{effect}}\right.$
$=8.62$ ). Thus, the interactions presumably reflect the effects of idiosyncratic stimulus items and will not be given any further consideration.

The data plotted in Figure 2 confirm the presence of a symbolic distance effect for judgments of color similarity. It can be seen that decision time generally decreased in a linear fashion as subtractive distance increased. Trend analysis of the distance main effect revealed a significant linear component $[F(1,32)=$ $\left.37.59, \mathrm{MS}_{\text {effect }}=.60, \mathrm{MSe}=.016, \mathrm{p}<.001\right]$, but no significant higher order components. A negative function was obtained for both the between- and withincategory comparisons, as well as for the directionality variable within these conditions. These distance effects are comparable to those obtained in other studies of symbolic comparison (see Banks, 1977; Moyer \& Dumais, 1978, for a review) and were obtained using a comparative dimension that should eliminate any effects of verbal response bias due to polar reference or end anchor effects.

Exceptions to the smoothness of the functions are found at subtractive distance Groups 2 and 5 for the unidirectional triplets. It can be seen that there is a rise and a sharp drop for these triplets at distance Groups 2 and 5 , respectively, in the between-category condition. Conversely, there is a drop at distance Group 2 for unidirectional triplets in the within-category condition. This opposite trend at distance Group 2 suggests again that the unidirectional fluctuations and the two resultant interactions already discussed are probably due to the effects of idiosyncratic items. ${ }^{2}$

The data in Figure 2 show no effect of directionality on RT. An analysis of error data, however, revealed a higher error rate for bidirectional (mean $=14.58 \%$ ) than for unidirectional $($ mean $=8.33 \%)$ triplets

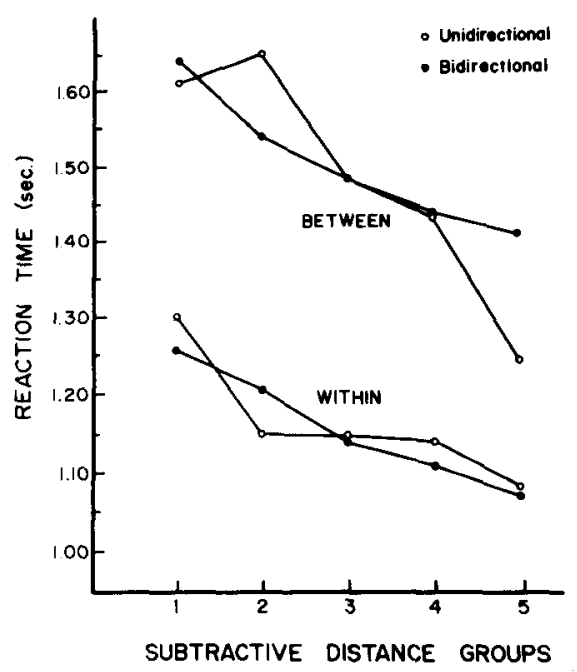

Figure 2. Median reaction times for color similarity comparisons for between- and within-color category triplets and for uniand bidirectional triplets as a function of subtractive distance group. 
$[\mathrm{t}(15)=4.63, \mathrm{p}<.001]$. This result indicates that comparison of similarities straddling the reference point (i.e., the color name) in bidirectional triplets is in some sense more difficult than comparison in unidirectional triplets. The increased difficulty of the former parallels findings in number comparison and preference judgments. Holyoak (1978) found that for unilateral and bilateral number triplets (e.g., the comparison Pairs 3-4 vs. 4-7 for reference point $=5$ ), RT to choose the digit closer to five as slower for bilateral than for unilateral triplets. Coombs (1958), in a preference judgment task, found that subjects produce more inconsistencies in their preference orderings of gray chips varying along the light-dark dimension if the chips lay on opposite sides of the "ideal" or maximally preferred chip than when the chips lay on the same side. There may be a number of reasons for the increased difficulty of bilateral cases, especially given that this difficulty was only manifested in the error data for the present experiment. Further research is needed here.

The data in Figure 2 show that comparisons involving a color chip within the category defined by the color name were consistently faster than comparisons made solely across color categories. This effect may be the result of matches among verbal labels for the colors in within-category triplets. Subjects may have named the chips implicitly and then responded rapidly whenever one of these labels matched the color name. Another verbalization strategy that could lead to faster within. category choices would be for subjects to choose the color chip that could not be easily named, since perripheral chips, in contrast to focal chips, are poorer examples of their color categories. Such strategies could account for the main effect of the within-category condition and, indeed, might be expected, since any time a peripheral color chip was seen it was the correct choice. However, what makes the within-category results interesting is that such verbalization strategies do not predict the distance effect found for these triplets. Why should subtractive distance have any effect on the speed of the match between identical verbal labels or the time needed to evaluate a color chip's nameability? Clearly, such strategies cannot solely account for the comparison process in within-category triplets.

A partial explanation of the main effect may lie in uncontrolled ratio or least distance differences between the between- and within-category comparison conditions. Examination of the previously calculated ratio distance measures revealed them to be generally larger for triplets in the between-category condition. For example, the red (chip)/green (name)/yellow (chip) triplet with a subtractive distance of 4 has a ratio of $11 / 15=.73$ between the original distances of the color chips from the color name. In contrast, the purple (chip)/pink (name)/peripheral pink (chip) with the same subtractive distance has a ratio of $2 / 6=.33$ between the original distance of the color chips from the color name.
Ratios for all triplets showed an average of .61 for between-category triplets and an average of .27 for within-category triplets. Similarly, the average least distance measure for between-category triplets was 9.01 , compared to an average of 2.21 for within-category triplets. To determine whether the between-/withincategory effect may have been caused by the effects of an uncontrolled distance measure, a regression analysis of RT with several indices of psychological distance was conducted.

\section{Other Distance Measures}

For each of the 160 unique triplets used in the four basic lists of the experiment, the three distance measures, subtractive, ratio, and least, were used. An additional dichotomous variable, B-W (one for betweencategory triplets, two for within-category triplets), was defined. These measures and the variable were to some extent intercorrelated, and the intercorrelation matrix is given in Table 2. Mean RTs were computed for each triplet. In computing mean RTs, only data obtained from trials where a correct response was made were considered.

Table 3 shows the intercept, slope, and $R^{2}$ values for each distance measure and the dichotomous variable, using the method of least-squares regression. Considering only the distance measures, the table shows that the greatest amount of variance in mean RTs is accounted for by least distance, then ratio distance, and to a lesser extent by subtractive distance. Thus, the latter seems to be a relatively poor predictor of RT when compared to ratio and least distance, although still significant $(p<.001)$. Because of the a priori intercorrelations among the distance measures, their relative predictive strengths warrant closer examination. Using the method of partial correlation, estimates can be made

Table 2

Intercorrelation Matrix for Distance Measures

\begin{tabular}{cccc}
\hline $\begin{array}{c}\text { Distance } \\
\text { Measures }\end{array}$ & Subtractive & Ratio & Least \\
\hline Ratio & -.764 & & \\
Least & -.311 & .761 & \\
B-W* & .201 & -.617 & -.739 \\
\hline
\end{tabular}

*Dichotomous variable $(I=$ between-category triplets, $2=$ within-category triplets).

Table 3

Intercept (Milliseconds/Unit), Slope (Milliseconds/Unit), and $\mathbf{R}^{2}$ Values for Distance Measures

\begin{tabular}{lcrc}
\hline $\begin{array}{l}\text { Distance } \\
\text { Measure }\end{array}$ & Intercept & \multicolumn{1}{c}{ Slope } & $\mathbf{R}^{2}$ \\
\hline Subtractive & 1671.14 & $-28.57^{*}$ & .131 \\
Ratio & 1104.93 & $766.96^{*}$ & .440 \\
Least & 1135.79 & $51.97^{*}$ & .553 \\
B-W & 2072.58 & $-438.31^{*}$ & .465 \\
\hline
\end{tabular}

${ }^{*} p<.001$ 
Table 4

Partial Correlations for Distance Measures

\begin{tabular}{llc}
\hline & \multicolumn{1}{c}{ Measure Removed } & $\mathrm{R}$ \\
\hline Subtractive & & $-.362^{* *}$ \\
Distance & Ratio & $.301^{* *}$ \\
Measure & Least & $-.205^{*}$ \\
& B-W & $-.314^{* *}$ \\
& Ratio, Least, B-W & -.118 \\
Ratio & & $.663^{* *}$ \\
Distance & Subtractive & $.643^{* *}$ \\
Measure & Least & $.224^{*}$ \\
& B-W & $.421^{* *}$ \\
& Subtractive, Least, B-W & -.003 \\
Least & & $.744^{* *}$ \\
Distance & Subtractive & $.712^{* *}$ \\
Measure & Ratio & $.493^{* *}$ \\
& B-W & $.487^{* *}$ \\
& Subtractive, Ratio, B-W & $.342^{* *}$ \\
B-W & & $-.682^{* *}$ \\
Distance & Subtractive & $-.667^{* *}$ \\
Measure & Ratio & $-.462^{* *}$ \\
& Least & $-.293^{* *}$ \\
& Subtractive, Ratio, Least & $-.295^{* *}$ \\
\hline & ( $p$ & \\
\hline
\end{tabular}

of the relation between a predictor and $\mathrm{RT}$ after the effects of other predictors have been removed. Table 4 presents the results of such an analysis.

Table 4 shows that subtractive distance accounts for only $9 \%$ of the variance in RT when ratio distance is partialled out, and $4 \%$ of the variance in RT when least distance is partialled out. Conversely, ratio and least distance still account for $41 \%$ and $51 \%$ of the variance, respectively, when subtractive distance is removed from each measure. Since ratio and least are better than subtractive distance as predictors of RT, some of the between-/within-category effect in Figure 2 may be explainable as a ratio or least distance effect. Such an interpretation would seem to fit nicely with the results of Hutchinson and Lockhead (1977), who found that decisions about which of two named animals was more similar to a third were faster when the comparison animals were from the same category as the reference animal than when they were from different categories. Nonetheless, part of the between-/within-category effect in Figure 2 must also be due to some unique property of within-category triplets, such as peripheral chips always representing the correct choice. This follows because the dichotomous variable in Table 4 remains a significant predictor of RT after the effects of all three distance measures are removed.

Tables 3 and 4 reveal that least distance is the strongest predictor of RT. This result is somewhat anomalous in the light of current knowledge about models of comparative judgment. At first glance the least distance effect would support a counting model such as that of Parkman (1971). For comparisons of color similarity, the model would work as follows. Upon presentation of a triplet, the subject might scan, starting at the color name, a memorial (image) representation of the color circle, possibly in both directions simultaneously (separate consideration of uni- and bidirectional triplets gave the same pattern of results as those presented in Tables 2, 3, and 4). When one of the comparison chips in a triplet is encountered in this process, the subject chooses it as the most similar and responds appropriately However, such a model predicts that no distance effect will remain once the effect of least distance is partialled out. Parkman himself and others have shown that this prediction does not hold up (Buckley \& Gillman, 1974; Shepard, Kilpatrick, \& Cunningham, 1975). The present data as well still show significant correlations for subtractive and ratio distance when the effect of least distance is removed (Table 4). Clearly, a pure counting or scanning model does not explain the comparison process in the present experiment.

A more likely account of the least distance effect may be made by considering the instructions given subjects. Subjects were asked to choose the more similar of two color chips to a color name. These instructions may set subjects to expect a cluster of highly similar colors. Presenting a triplet in accord with subjects' expectations would permit a faster response than a triplet violating expectations. Such an expectancy hypothesis has recently been proposed to account for semantic congruity effects in symbolic comparison tasks (Marschark \& Paivio, in press). With this expect. ancy account of the least distance effect, a discriminability measure more in accord with current data could still be used to predict decision time in the present task. Distance ratios, for example, predict that RT (1) increases monotonically with the distance of the comparison items from the reference point and (2) decreases monotonically as the difference between the two items increases. For the present data, the increase in RT in Prediction 1 above could in part be due to a violation of expectation engendered by the instructions. If so, a test of this hypothesis would be to present subjects with the same color comparison task but with instructions to choose the more dissimilar color chip. The prediction from the expectancy hypothesis is that the increase in RT in Prediction 1 above would be somewhat attenuated (but not eliminated, since it is the distance of the comparison items from the reference point together with the distance between the comparison items that determines the ratio value), since subjects would now be prepared for a cluster of dissimilar colors.

A word of caution is important. The present experiment was designed solely for the purpose of examining whether subtractive distance would produce a symbolic distance effect in color comparison, and not as a means for evaluating the predictive power of several distance measures. The preceding analysis was conducted primarily to determine whether the between-/withincategory effect in Figure 2 could be attributable to the 
effects of uncontrolled distance measures. The results, in part, confirm this premise. However, the results of future research in color comparison testing predictions from the expectancy hypothesis or examining the predictive strengths of several distance measures would not affect the general conclusions from this study. Briefly stated (and discussed in more detail below), the symbolic distance effect, because it was obtained using colors around the hue circle and a triplet paradigm of comparison, provides compelling support for an analog model of symbolic judgment.

\section{DISCUSSION}

The present research clearly demonstrated a symbolic distance effect for comparison of color similarity. The effect was found to be consistent over subtractive distances obtained through unidirectional and bidirectional measurement around the hue circle, and for comparisons between and within color categories. Although the correlational analysis indicated that subtractive distance is not the best predictor of RT, the overriding significance of the distance effects obtained is the support they provide for an analog model of comparative judgment. If the positions of Katz and Postal (1964), that color distinctions are not codable by linguistic features, and of Shepard (1964), that colors tend to be regarded as "unanalyzable wholes," are accepted, then it is difficult to explain how the similarity comparisons could be made other than through a "psychophysical" comparison of color chips and an analog (image) representation of the color name on the hue circle. The memorial data base necessary for any discrete (propositional, feature, etc.) semantic memory theory to explain the symbolic distance effect would not exist for color.

This is also true for a semantic coding model of symbolic comparison (Banks, 1977; Banks, Clark, \& Lucy, 1975; Banks \& Flora, 1977). This model assumes that a discrete linguistic code is associated with the stimuli in a comparative judgment task (e.g., "small" and "large" for two stimuli on the size dimension) that are then compared and matched to the instructions so that a response can be made. However, these codes are assumed to be generated from a data base that stores information about individual items as propositions or feature elements of some sort (Banks, 1977). Such a data base would not exist for representing individual colors. The semantic coding model could assume that information about the relationships (e.g., similarity) between colors are stored as linguistic codes in longterm memory. Thus, for red the code "very similar" may relate it to orange, the code "similar" may relate it to yellow, and so on. This idea, while plausible, becomes rather unparsimonious when held to account for the present results, since it would require that all perceptually discriminable colors (both across and within categories, since a significant distance effect was ob- tained for within-category triplets) be coded relative to all other colors in this way. That subjects would retain a large linguistic structure of relational information in this manner seems unlikely. Furthermore, the number of finely-graded distinctions between colors would require that the linguistic codes relating them also be finely graded (e.g., similar, similart, similart+, etc.). But, as Moyer and Bayer (1976) have pointed out, such modifications of semantic code theories cause them to resemble an analog model.

An additional problem with the semantic coding model arises from the fact that certain stimuli in the present experiment were presented in analog form (i.e., as color chips). The semantic coding model assumes that such stimuli must be translated from their analog forms into digital codes (Banks et al., 1975, p. 36). This translation process, which precedes the critical comparison/decision stage, is assumed to produce "coded semantic descriptions." It is hard to conceive of the form that these descriptions would take in the case of color stimuli. If these codes are merely similarity labels (such as "very similar" and "similar" relative to the variable color name reference point), then the semantic coding model simply begs the question regarding the basis and stage at which colors are compared.

Finally, it could be argued that subjects made their comparisons on the basis of verbal associations between color names. Names do not contain explicit hue similarity information, but there do exist verbal associative habits between adjacent colors on the hue circle. Examples are "greenish blue" and "reddish orange." In some cases subjects may have chosen the more similar color chip on the basis of such associations, but such a strategy could not explain the choices made for those triplets where the color chips were not adjacent, and hence would not possess verbal associations with the color name. It seems, then, that the best account of color similarity comparison and the RT functions obtained in this experiment is in terms of a perceptual analog model.

In summary, the results of this experiment demonstrated that a symbolic distance effect can be obtained in comparison of color similarity. The effect was obtained for various conditions of comparison and over a unique comparative dimension, the hue circle, using a triplet paradigm. These findings impose serious constraints on long-term memory models that assume a discrete (essentially linguistic) data base for symbolic comparisons, since colors appear to be items that do not readily lend themselves to feature coding in memory. The results are consistent with models that allow symbolic stimuli to be compared on the basis of analog codes in memory.

\section{REFERENCE NOTE}

1. Shepard, R. N., \& Cooper, L. A. Representation of colors in normal, blind, and color-blind subjects. Paper presented at 
the joint meeting of Division 5 of the American Psychological Association and the Psychometric Society in Chicago, 1975.

\section{REFERENCES}

AttNeave, F. Representation of physical space. In A. W. Melton \& E. Martin (Eds.), Coding processes in human memony. New York: Winston-Wiley, 1972.

Banks, W. P. Encoding and processing of symbolic information in comparative judgments. In G. H. Bower (Ed.). The psychology of learning and motivation (Vol. 11). New York: Academic Press, 1977.

Banks, W. P., Clark, H. H., \& Lucy, P. The locus of the semantic congruity effect in comparative judgments. Journal of Experimental Psychology: Human Perception and Performance, $1975,1,35-47$.

Banks, W. P., \& Flora, J. Semantic and perceptual processes in symbolic comparisons. Journal of Experimental Psychology: Human Perception and Performance, 1977, 3, 278-290.

BerLIN, B., \& KAY, P. Basic color terms: Their universality and evolution. Los Angeles: University of California Press, 1969.

Buckley, P. B., \& Gillman, C. B. Comparisons of digits and dot patterns. Journal of Experimental Psychology, 1974, 103, 1131-1136.

Carroll, J. D., \& Criang, J. J. Analysis of individual differences in multidimensional scaling via an $\mathrm{N}$-way generalization of "Eckart-Young" decomposition. Psychometrika, 1970, 35, 283-319.

Coomss, C. H. On the use of inconsistency of preferences in psychological measurement. Journal of Experimental Psychology, 1958, 55, 1-7.

Cooper, L. A., \& Shepard, R. N. Chronometric studies of the rotation of mental images. In W. G. Chase (Ed.), Visual information processing. New York: Academic Press, 1973.

Curtis, D. W., Paulos, M. A., \& Rule, S. J. Relation between disjunctive reaction time and stimulus difference. Journal of Experimental Psychology, 1973, 99, 167-173.

Farnsworth-Munsell 100-hue test for the examination of color discrimination. Baltimore Md: Munsell Color Company, Inc., 1957.

Fillenbaum, S., \& Rapoport, A. Structures in the subjective lexicon. New York: Academic Press, 1971.

Holyoak, K. J. Comparative judgments with numerical reference points. Cognitive Psychology, 1978, 10, 203-243.

Hutchinson, J. W., \& Lockhead, G. R. Similarity as distance: A structural principle for semantic memory. Journal of Experimental Psychology: Human Learning and Memory, 1977, 3, 660-678.

Katz, J. J., \& Postal, P. M. An integrated theory of linguistic descriptions. Cambridge, Mass: MIT Press, 1964.

Marschark, M., \& Paivio, A. Semantic congruity and lexical marking in symbolic comparisons: An expectancy hypothesis. Memory \& Cognition, in press.

MOYER, R. S. Comparing objects in memory: Evidence suggesting an internal psychophysics. Perception \& Psychophysics, 1973, 13, 180-184.

Moyer, R. S., \& BAYER, R. H. Mental comparisons and the symbolic distance effect. Cognitive Psychology, 1976, 8, 228-246.

Moyer, R. S., \& Dumais, S. T. Mental comparison. In G. H. Bower (Ed.), The psychology of learning and motivation (Vol. 12). New York: Academic Press, 1978.
Moyer, R. S., \& Landauer, T. K. The time required for judgments of numerical inequality, Nature, 1967, 215, 1419-1520.

Munsell Color Company. The Munsell book of color Glossy finish collection. Baltimore, Md: Author, 1966.

Paivio, A. Perceptual comparisons through the mind's eye. Memory \& Cognition, 1975, 3, 635-647.

PaIvio, A. Imagery, language and semantic memory. International Journal of Psycholinguistics, 1978, 5, 27-43.

Parkman, J. M. Temporal aspects of digit and letter inequality judgments. Journal of Experimental Psychology, 1971, 91, 191-205.

SHEPARD, R. N. Attention and the metric structure of the stimulus space. Journal of Mathematical Psychology, 1964, 1, 54-87.

Shepard, R. N., Kilpatrick, D. W. \& Cunningham, J. P. The internal representation of numbers. Cognitive Psychology, 1975, 7, 82-138.

\section{NOTES}

1. For the purpose of the present study, the above measures were taken to differentiate colors primarily in terms of hue, although some brightness variation is included in the measure. Not included in the measures are slight differences in saturation between colors. For two reasons this confounding does not seriously restrict conclusions from the study. First, in all but four of the above measures, the distance for hue is greater than that for both brightness and saturation. The exceptions are the distances between the chips for focal red and orange, focal purple and pink, focal orange and peripheral (b) orange, and focal pink and peripheral (b) pink, where hue and brightness contribute equally to the measure. Second, it seems that brightness and saturation variation may play little or no role in similarity comparison of named colors. Fillenbaum and Rapoport (1971) had subjects rank order all possible pairs of 15 color names on the basis of subjective similarity. In a scaling analysis of the data, they found that the color name space yielded an orderly array of almost perfect correspondence to the hue circle, but found little effect of brightness or saturation, although certain color names varied considerably on these dimensions. Also, in the present experiment, a scaling analysis (INDSCAL, Carrol \& Chang, 1970) performed on subjects' choices for each triplet yielded a two-dimensional array of close correspondence to the hue circle, with little effect of brightness or saturation. Such results indicate that brightness and saturation information has minimal weight in comparison of memory color similarity, although the memory representations may contain such information.

2. Trend analysis of the data plotted in Figure 2 revealed the presence of a significant quadratic component $[F(1,32)=6.5$, $\mathrm{MS}_{\text {effect }}=.13, \mathrm{MSe}=.02, \mathrm{p}=.03 \mathrm{]}$ in the distance function for unidirectional triplets in the between-category condition. Since this was the only function possessing a significant higher order component, probable idiosyncrasies in the stimulus items for this condition are felt to provide the best explanation for the higher order trend and the resultant interactions.

(Received for publication July 25, 1978; revision accepted February $7,1979$. 